\title{
Buruli ulcer: wound care and rehabilitation
}

This article was published in the following Dove Press journal:

Chronic Wound Care Management and Research

27 June 2016

Number of times this article has been viewed

\section{Michael Frimpong' \\ Fred Stephen Sarfo \\ Mabel Sarpong Duah' \\ Mark Wansbrough-Jones ${ }^{3}$ \\ Richard O Phillips ${ }^{2}$ \\ 'Kumasi Centre for Collaborative Research in Tropical Medicine, ${ }^{2}$ Department of Medicine, School of Medical Sciences, Kwame Nkrumah University of Science and Technology, Kumasi, Ghana; Institute for Infection and Immunity, St George's University of London, London, UK}

Correspondence: Richard O Phillips Department of Medicine, School of Medical Sciences, Kwame Nkrumah University of Science and Technology, University Post Office, PMB, Kumasi, Ghana

Email rodamephillips@gmail.com

\begin{abstract}
Buruli ulcer caused by Mycobacterium ulcerans is a neglected tropical disease characterized by extensive ulceration involving predominantly the upper and lower limbs of patients. The disease is common in rural tropical communities in West and Central Africa, where access to proper health care is limited. Pathogenesis of the characteristic painless ulcers is linked to the elaboration by M. ulcerans of a lipid toxin called mycolactone that has potent cytopathic, immunosuppressive, and analgesic effects on a host of cells in cutaneous tissues. Mycolactone is known to profoundly inhibit secretion of a plethora of proteins that are essential for wound healing. Even though a combination antibacterial therapy of streptomycin and rifampicin for 8 weeks is effective for treatment, it relies on good and appropriate wound management to prevent secondary bacterial infections and improve healing. Evidence-based interventions for wound care in Buruli ulcer disease are often lacking and have relied on expert advice and recommendations. Surgical interventions are limited to debridement of necrotic tissue and grafting of extensive ulcers, usually after antibiotic therapy. Patients' rehabilitation is an important component of care to reduce disabilities associated with the disease and proper integration into the community after treatment.
\end{abstract}

Keywords: Buruli ulcer, Mycobacterium ulcerans disease, Mycobacterium ulcerans, wound care, rehabilitation, disability

\section{Introduction}

Buruli ulcer (BU) is a chronic, necrotizing skin disease caused by infection with a slow growing pathogen, Mycobacterium ulcerans. ${ }^{1,2}$ M. ulcerans is currently the third most common mycobacterial pathogen of humans, after Mycobacterium tuberculosis and Mycobacterium leprae, which cause tuberculosis and leprosy, respectively. A large proportion of the BU cases are reported by 33 countries from tropical, subtropical, and temperate climates in Africa, South America, and the Western pacific regions, respectively. The majority of the cases reported from sub-Saharan Africa are from poor rural communities. ${ }^{1,3}$ Even though there is a strong association of the occurrence of the disease with stagnant or flowing water bodies, the specific mode of transmission seems elusive. ${ }^{4,5}$ The disease usually manifests itself as a painless nodule, a firm plaque, or an edematous lesion, which soon ulcerates with characteristic undermined edges. ${ }^{1,3,6}$ BU may also present as osteomyelitis. ${ }^{1,3,7}$ Treatment of BU disease (BUD) has evolved over the past decade, leading to the use of antibiotics as replacement to surgery. The combination of rifampicin and streptomycin daily for 8 weeks is effective in healing all forms of lesions caused by M. ulcerans, and this has reduced the submit your manuscript | www.dovepress.com Dovepress http://dx.doi.org// 0.2147/CWCMR.S85469 
recurrence rate from $6 \%-47 \%$ after surgery to $0 \%-2 \%$ after antibiotic treatment. ${ }^{8}$ Despite antibiotic efficacy with healing of up to two-thirds of patients within 25 weeks after initiation of treatment, the time to healing differs significantly for similar-sized lesions, ${ }^{9-11}$ and this is influenced by the quality of wound care. As ulcerative disease forms the majority of lesions in many treatment centers, ${ }^{7-9,12}$ wound care plays a pivotal role in the care and treatment outcomes of patients. ${ }^{13}$ In addition, as a result of late presentation to health facilities, scarring and contractures may complicate the healing process, particularly among patients who are not treated early, leading to long-term functional disability and occasionally amputation of affected limbs. ${ }^{1,14}$ In this article, we review the current knowledge on BU with particular emphasis on wound care and rehabilitation.

\section{Epidemiology and transmission}

The main burden of disease falls on children living in subSaharan Africa, but healthy people of all ages, races, and socioeconomic classes are susceptible. Most cases are from West Africa, notably Benin, Cote d'Ivoire, and Ghana. ${ }^{15}$ Cote d'Ivoire is the most affected country reporting $>2,500$ cases per annum. Every year, 5,000-6,000 cases are reported to the World Health Organization (WHO) from 15 of the 33 participating countries, although there is considerable underreporting ${ }^{1,16}$

BU has been referred to as a mysterious disease because the exact mode(s) of transmission remains unclear, although several hypotheses have been proposed. The disease has been widely associated with proximity to aquatic habitats. A conceptual model of $M$. ulcerans transmission initially propounded by Portaels et $\mathrm{al}^{17}$ and expanded on by Merritt et $\mathrm{al}^{18}$ and Marion et $\mathrm{al}^{19}$ has been posited as follows: $M$. ulcerans present in mud, detritus, water filtrants, and plant biofilms can be picked up and concentrated in grazing or filtering aquatic insects (eg, midges and mosquito larvae) or other invertebrates (snails, crustaceans, plankton) through their feeding activities. Then, predatory aquatic vertebrates (ie, some fish) and invertebrates (eg, true bugs, beetles, and dragonfly larvae) feed on other invertebrate prey or small fish, serving to move $M$. ulcerans from prey to biting insects. Finally, aquatic insects capable of flight and birds that prey on fish and/or aquatic invertebrates may potentially disseminate $M$. ulcerans to other aquatic environments. ${ }^{18}$ Within this model, humans may come into contact with $M$. ulcerans via several potential vectors through their activities in endemic communities. In Australia, elderly people living in retirement homes by the sea are susceptible to BU. Here, the ring-tailed possoms are often infected and develop ulcers around the mouth and nose. ${ }^{20,21}$ These animals live in the trees and have no contact with humans, but since mosquitoes ${ }^{22}$ in such places also carry $M$. ulcerans DNA, it has been hypothesized that they are involved in the cycle of transmission. However, this has been difficult to prove. Due to limited understanding of the vector and mode of transmission, control efforts are directed toward early case detection and treatment.

\section{Clinical presentation}

M. ulcerans disease (BU) manifests as a painless skin nodule, papule, or plaque, which may be associated with a rapidly expanding area of edema. All these early lesions progress to central ulceration with undermined edges over a variable period of weeks (Figures 1 and 2). ${ }^{23}$ Lesions may occur at any location, but a predilection for affecting limbs has been observed. Interestingly, in spite of the presence of large ulcers, patients usually have no systemic symptoms, which together with the painless nature of ulcers may explain why sufferers seek medical help late. At advanced stages of the
A

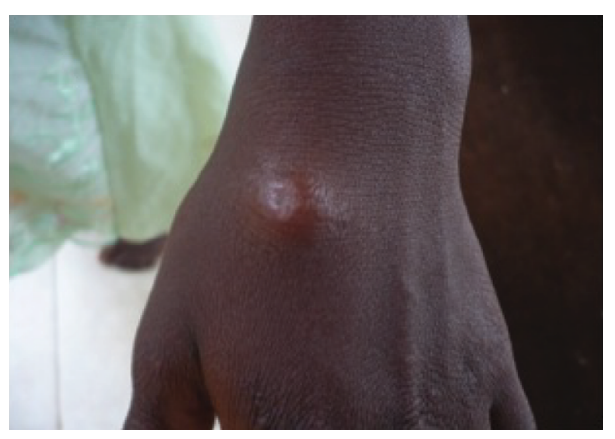

B

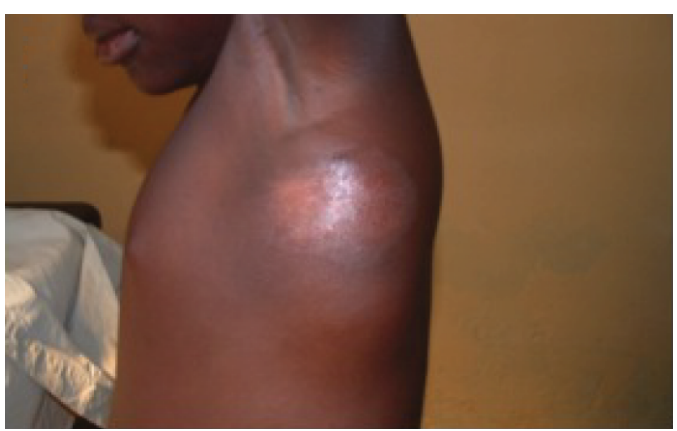

C

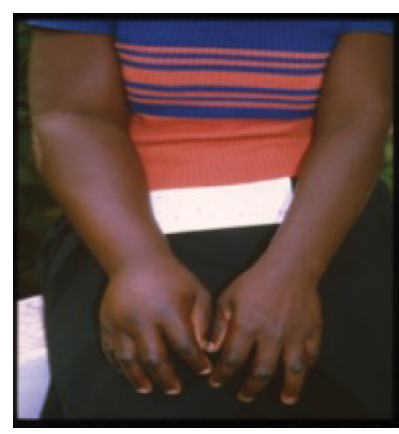

Figure I Non-ulcerative forms of BU of $(\mathbf{A})$ the left hand showing a nodule, $(\mathbf{B})$ the trunk showing a plaque, (C) the right upper limb showing the edematous form. Note: Courtesy of BU photo library of Agogo Presbyterian Hospital, Agogo, Ghana.

Abbreviation: BU, Buruli ulcer. 
A

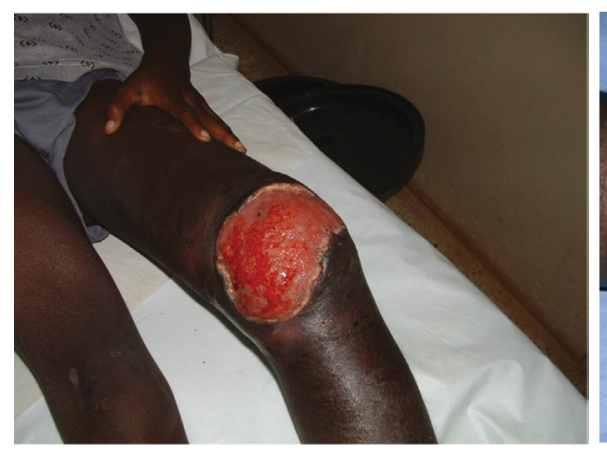

B

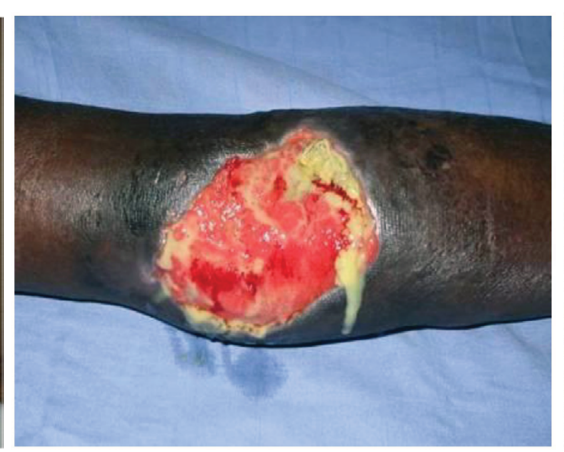

C

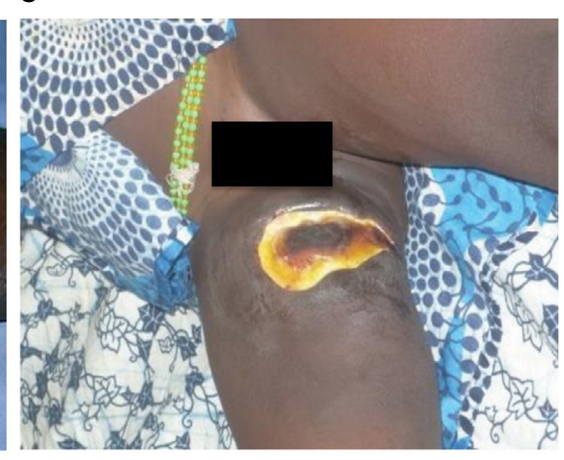

Figure $2 \mathrm{BU}$ of (A) the knee with granulation, (B) the left knee with yellow slough, and (C) the right thigh with black necrotic tissue.

Note: Courtesy of BU photo library of Agogo Presbyterian Hospital, Agogo, Ghana.

Abbreviation: $\mathrm{BU}$, Buruli ulcer.

disease, osteomyelitis may develop in association with BU. ${ }^{24}$ Severity of lesions is assessed using the widest diameter of lesions: category I $<5 \mathrm{~cm}$; category II between $5 \mathrm{~cm}$ and $14.9 \mathrm{~cm}$; category III $>15 \mathrm{~cm}$. Notably, category III also includes multiple lesions and those involving critical areas such as the genitals or the face. ${ }^{25}$

\section{Diagnostic confirmation}

Laboratory confirmation of a clinical diagnosis of $M$. ulcerans disease is currently performed using either of the following: culture for M. ulcerans, staining for acid-fast bacilli, or polymerase chain reaction (PCR) for IS2404 or histology. Smear microscopy for detection of acid-fast bacilli is the simplest diagnostic technique available in endemic communities in sub-Saharan Africa. Swabs from the undermined edges of an ulcer or biopsies obtained surgically or by punch biopsy are used to prepare a smear for Ziehl-Neelsen staining. ${ }^{26}$ This has an average sensitivity of $~ 50 \%(29 \%-78 \%) .^{27-29}$ Higher sensitivity is achieved with surgical samples, being larger, but minimally invasive techniques are required to obtain samples for laboratory confirmation. Microscopic examination has a high specificity of up to $96.6 \% ;^{28}$ skin infection with other mycobacteria is uncommon in rural areas.

Culture for M. ulcerans on Lowenstein-Jensen slopes takes 9-12 weeks on average but it can be up to 6 months, and its sensitivity ranges from $34 \%$ to $79 \%{ }^{28}$ In addition to its low sensitivity, the main drawback with culture as a diagnostic technique is the time it takes to obtain a positive result. This constitutes a challenge for both initiating antibiotic therapy and knowing whether infection has been eliminated.

At a sensitivity of $82 \%$ in a Ghanaian study, ${ }^{27}$ histology was perhaps the most sensitive of the conventional diagnostic methods before the advent of PCR for IS2404. However, histopathology expertise is not often available in endemic areas of Africa.

A major advancement for the purpose of clinical management and research studies is the application of PCR for the insertion sequence $I S 2404,{ }^{30}$ one of the two multicopy insertion sequences found in the genome of M. ulcerans, ${ }^{31}$ to swabs or tissue samples..$^{27,28,31-33}$ With an analytical specificity of $100 \%$ and a sensitivity of $79 \%-98 \%, I S 2404$ PCR is considered to be the most reliable technique for the detection of $M$. ulcerans in human diagnostic samples. These sensitivity and specificity data for PCR are from studies using punch biopsies or swabs. Recently, fine-needle aspirates obtained from lesions have been used for PCR diagnosis of $M$. ulcerans disease with a sensitivity of up to $90 \% \cdot{ }^{29}$ Hence, even though the sensitivity of PCR is slightly compromised with fineneedle aspirate samples, its acceptance and tolerability by patients have pushed it into the first-line sampling strategy for non-ulcerative lesions. ${ }^{34,35}$

\section{Pathogenesis of $\mathbf{M}$. ulcerans disease}

$M$. ulcerans causes a subcutaneous infection, which is often described as a panniculitis, but histological examination typically shows large clumps of extracellular acid-fast organisms surrounded by areas of necrosis and a poor or an absent inflammatory response. ${ }^{36}$ The histological hallmark of M. ulcerans infection is that despite extensive necrosis there is minimal evidence of an initial acute inflammatory response. In contrast to other pathogenic mycobacteria that are facultative intracellular parasites of macrophages, $M$. ulcerans occurs in lesions primarily as extracellular clumps and the necrosis extends some distance from the site of bacterial colonization. This led to the hypothesis that $M$. ulcerans secretes an exotoxin. ${ }^{37}$

In 1999, a significant breakthrough occurred in the purification and isolation of the proposed toxin from the 
acetone-soluble lipid fraction of extracts from M. ulcerans bacterial pellets. ${ }^{38}$ This lipid toxin designated as mycolactone was a polyketide-derived macrolide and was shown to produce cytopathicity on cultured L929 murine fibroblasts and cell cycle arrest in the $\mathrm{G}_{0} / \mathrm{G}_{1}$ phase of the cell cycle. Furthermore, intradermal inoculation of purified toxin into guinea pigs produced a lesion similar to that of BU in humans. The lipid toxin was called mycolactone to reflect the mycobacterial source and chemical structure. This exciting piece of work paved the way for subsequent studies into the role of mycolactone in the pathogenesis of $M$. ulcerans disease.

Mycolactone is composed of an invariant 12-membered lactone ring to which two polyketide-derived, highly unsaturated acyl side chains are attached. It is synthesized by a $174 \mathrm{~kb}$ megaplasmid named pMUM001, which has three very large genes ( $m l s A 1: 51 \mathrm{~kb}, m l_{s} A 2: 7 \mathrm{~kb}$, and $m l_{s B}: 42 \mathrm{~kb}$ ) that encode the modular type I polyketide synthases. ${ }^{39}$ There are variations in the type or congeners of mycolactone produced, depending on the origin of strains of $M$. ulcerans. For instance, pathogenic human M. ulcerans strains from Africa, Australia, and the People's Republic of China predominantly produce mycolactone A/B $(m / z 765), \mathrm{C}(\mathrm{m} / z$ 749), and D $(\mathrm{m} / z$ 779), respectively, with each strain producing minor quantities of other congeners. ${ }^{40}$ By far, the most potent biologically active congener of mycolactone is the mycolactone A/B produced by the African strains. ${ }^{40}$

It is now believed that the pathogenesis of M. ulcerans disease is essentially mediated by the elaboration of mycolactone at the site of infection. The extensive tissue necrosis and minimal inflammation in BUs constitute the hallmarks of these lesions and reflect the cytocidal and immunosuppressive properties of this toxic macrolide. Being a lipid molecule, mycolactone is rapidly diffusible across plasma membranes of cells at the site of infection and accumulates within the cytosol. Mycolactone then triggers diverse cytopathic effects, including cytoskeletal rearrangements and cell cycle arrest, eventually culminating in apoptotic or necrotic cell death. ${ }^{41}$ In adherent cells such as fibroblasts and epithelial cells, mycolactone targets scaffolding proteins such as Wiskott-Aldrich syndrome protein that controls actin dynamics leading to cell detachment and death by a process referred to as "anoikis", which probably underlies ulcer formation in BUD. ${ }^{42}$ The manifestations and extent of mycolactone-mediated cytotoxicity vary considerably among cell types, suggesting that the molecular target of mycolactone may be differentially expressed or have different functions in different cells. At lower, noncytotoxic concentrations, mycolactone displays immunomodulatory properties on human primary monocytes and dendritic cells, indicating that it may limit the initiation of innate immune responses in vivo. ${ }^{43,44}$ By blocking the capacity of primary T-cells to produce multiple cytokines upon activation and by impairing T-cell migration and homing into lymph nodes, mycolactone significantly inhibits the development of adaptive immune responses. ${ }^{45,46}$ Recent evidence has revealed that mycolactone exerts a profound effect on protein secretion by blocking the co-translational translocation of a plethora of proteins that pass through the endoplasmic reticulum for secretion or placement in cell membranes. ${ }^{47,48}$ It is noteworthy that many of these proteins are important in wound healing, and we hypothesize that the rate of healing of ulcers under antibiotic treatment may be significantly impacted by the rate of clearance of mycolactone from tissues. Studies are underway to evaluate the synergy between tissue levels of mycolactone, bacterial load, and rate of wound healing in BUD in humans. The central role of mycolactone in the pathogenesis and its potential application as a biomarker for BU has been reviewed by our group. ${ }^{49}$

\section{Management}

The introduction of antibiotic therapy for the management of BUD represents a significant advance in the care of patients with this disease. Currently, combination antimycobacterial therapy is the mainstay of treatment with additional treatment and care, where it is necessary with surgery and early basic wound management with appropriate dressings as well as physiotherapy when an ulcer is close to a joint. These additional interventions can mitigate and minimize complications such as contractures and facilitate timely return to normal activity. In countries such as Australia where surgery is readily available, excision with or without grafting is applied more frequently in conjunction with antibiotics.

\section{Antibiotic therapy}

The exciting prospects for utilization of antibiotics in the management of BUD were founded on the demonstration of sensitivity of $M$. ulcerans to rifamycins, ${ }^{50}$ aminoglycosides, ${ }^{51}$ macrolides, ${ }^{52}$ and some quinolones ${ }^{51}$ both in vitro and in vivo. ${ }^{53-55}$ In a mouse footpad model of infection, the best results were obtained with the combination of a rifamycin and an aminoglycoside, and importantly resistance to rifampicin was observed when it was used as monotherapy. ${ }^{53,56}$

The recommended antibiotic treatment is daily rifampicin $10 \mathrm{mg} / \mathrm{kg}$ orally and streptomycin $15 \mathrm{mg} / \mathrm{kg}$ intramuscularly for 8 weeks. This has been shown to result in healing of all forms of $M$. ulcerans disease with few exceptions. ${ }^{8,57}$ The 8 -week duration of treatment recommended by the WHO 
expert advisors after a study showed that $M$. ulcerans organisms were still viable in early lesions excised after treatment for 2 weeks, whereas cultures were sterile when treatment was extended to 4 weeks, 8 weeks, or 12 weeks. ${ }^{6}$

Further studies on antibiotic therapy are in progress. A continuing aim is to design an antibiotic regimen in which no injection is required. Recently, a controlled trial has shown that clarithromycin can be substituted for streptomycin in the second 4 weeks of treatment without loss of efficacy. ${ }^{58} \mathrm{~A}$ small observational study in Ghana also showed no difference in outcome when rifampicin and streptomycin were given for only 2 weeks followed by rifampicin and clarithromycin for 6 weeks. ${ }^{59}$ Importantly, a landmark trial is in progress to compare oral treatment with rifampicin and clarithromycin daily for 8 weeks with the currently recommended combination. An additional option if clarithromycin is not tolerated is moxifloxacin combined with rifampicin. ${ }^{60}$

\section{Antibiotic treatment complications}

The most common complication of antibiotic treatment is paradoxical reaction occurring during or after treatment in $8 \%-12 \%$ of patients. ${ }^{8,11,59,61-63}$ This is important because it can be confused with recurrence. Paradoxical reaction is defined as an episode of new inflammation, with or without pus formation, with significant enlargement of a healing lesion during or after antibiotic treatment. Paradoxical reaction usually occurs at the site of the original lesion but sometimes it occurs in a different area. It must be distinguished from apparent enlargement of an ulcer during the first 2 weeks of antibiotic treatment resulting from auto-debridement of necrotic tissue at the periphery of the ulcer. Paradoxical reaction is believed to be due to an immunological response to residual $M$. ulcerans antigens which are known to persist for many months after successful treatment. These reactions resolve without treatment other than completion of the standard 8-week course of antibiotics. There is no need to give additional antibiotics when the initial course has been completed as cultures are sterile, and there is no evidence that corticosteroid treatment is beneficial. ${ }^{8,59,64}$ The immunological mechanism underpinning paradoxical reactions requires clearer elucidation in order to design appropriate evidencebased interventions for this important clinical phenomenon.

When lesions are excised without antimycobacterial therapy, recurrent $M$. ulcerans infection occurs in 7\%-16\% but recurrence following antibiotic therapy alone is now known to be rare, only $0 \%-2.5 \%,{ }^{8,57,59}$ and many so-called recurrences in the past may have been paradoxical reactions. The reasons for recurrence are not known but lack of adherence to the antibiotic regimen may play a part. Importantly, development of antimicrobial resistance has not been reported in humans.

After a decade of successful antibiotic therapy with the WHO-recommended regimen made up of daily oral rifampicin together with intramuscular streptomycin for 8 weeks, ${ }^{3,8}$ side effects of medication have been examined. The use of streptomycin for long periods has been reported to cause ototoxicity and nephrotoxicity ${ }^{65-67}$ damaging both the cochlea and the vestibulum ${ }^{65,68}$ particularly in the elderly. ${ }^{67}$ We have found that the combination of rifampicin and streptomycin is safe and potential side effects, including skin rash (streptomycin and rifampicin), deafness, dizziness, decreased urine output (streptomycin), anorexia, nausea, abdominal pain, jaundice, and renal failure (rifampicin), are rare overall, occurring in $<2 \%$ of patients. ${ }^{57,58}$ In routine practice, patients who develop mild adverse effects are advised to continue treatment with appropriate management of the symptoms and close monitoring by health care providers. Moderate adverse effects may require temporary discontinuation of treatment or adjustment of the dosage with treatment discontinuation and referral to a hospital for further evaluation when side effects persist. It is notable that moderate and severe adverse events are seldom observed in our practice.

Sometime in the past, large ulcers on the limbs that have been neglected for a long time before presentation have ultimately required amputation, but this is now a rarity. Fortunately, lesions on the face and trunk are less common than those on the limbs ${ }^{8,57}$ but when they occur close to the eye there may be loss of vision and, on the trunk, the breasts or genitalia may be damaged. Lesions left untreated may undergo malignant transformation to an aggressive ulcerating squamous cell carcinoma known as Marjolin's ulcer. Increased public education through outreach programs has shown tremendous improvement in disease outcome.

\section{Wound care}

There are limited data on best wound care practice for $\mathrm{BU}$, and the current guidance is based on expert advice. ${ }^{69}$ Although there is lack of evidence, it is likely that good wound care with the right choice of dressing and a rational approach in applying topical solutions will reduce time to healing, pain, and morbidity. ${ }^{70}$

Basic principles of wound management involve treating or managing relevant systemic conditions, maintaining a moist wound environment, protecting the wound from trauma, promoting a clean wound base, and controlling infection, edema, and lymphedema. ${ }^{71}$ Currently, there are guidelines on BU wound care, published by the WHO, which apply 
the basic principles of general wound care to BU. ${ }^{72}$ Little is known about field practices relating to application of the basic principles as described in the WHO guidelines. In these guidelines, wound care is described in a step-by-step manner: assessment of the wound, preparation of the wound bed, and dressing of the wound. Assessment of the wound is based on the "red-yellow-black system", as described in the WHO prevention of disabilities manual. ${ }^{69}$ This system is simple, and it provides information on the phases of healing and basic principles of care required for each stage.

For the inflammatory phase when the wound color is pale pink to deep dark red, cleaning with $0.9 \%$ saline solution is proposed for cleaning the wound. For the proliferative phase when the wound is yellow or ivory in color with the tendency to slough and exude, $0.9 \%$ saline solution cleaning, debridement, and use of absorbent wound covering are advised. In the proliferative phase when the wound is black or brown with dead tissue and dry, $0.9 \%$ saline solution cleaning, debridement of eschar, and use of absorbent wound covering and moistening are advised. When there is dead tissue, the best approach is gentle spraying of saline solution onto the wound until most or all of the dead tissue is removed. ${ }^{69}$ Topical antiseptics are to be avoided, as these can damage newly forming epithelial and fibroblast cells. ${ }^{73-76}$ Debridement is ideally performed selectively, removing maximum amounts of dead tissue but minimizing damage to healthy tissue. Topical agents such as povidone iodine, sodium hypochlorite solution, acetic acid solution and hydrogen peroxide, potassium permanganate, methylrosanilinium chloride (gentian violet), mercurochrome, and merthiolate are generally not advised because of their potential harmful effects on tissues. ${ }^{69,76-78}$ Topical antibiotics are believed to have limited effectiveness, and they should be avoided in view of their tendency to encourage development of resistance.

The type of dressing applied is determined by the amount of exudate. A suitable wound dressing in resource-poor rural settings should be cheap, easy to apply, and not cumbersome for the patient. In this environment, the standard dressing is gauze coated with vaseline which is changed daily. Vaseline reduces the chance that the gauze will stick to the wound bed causing damage when the dressing is removed. ${ }^{13}$ If the gauze does stick to the wound bed, saline solution is applied until the gauze is freed easily. Wounds are covered to protect them from infection, injury, and drying out. ${ }^{69}$ Recent evidence from Ghana suggests that the cost of wound dressing supplies contribute substantially to the $\$ 570$ mean cost of BU treatment and compliance to standard care was dependent on support schemes. ${ }^{79}$
Many different products have been proposed to improve wound healing in BU. ${ }^{6}$ Highly absorbent dressings may have advantages in the early stages of treatment when there is excess necrotic tissue and exudate in the ulcer, but these are not always available. Recently, we have used Drawtex, a highly absorbent dressing that uses dispersion technology, acting through capillary action, hydroconductivity, and electrostatic action. It is reported to facilitate the removal of dead tissue, decreasing the bacterial load as well as clearing potentially harmful matrix metalloproteinase, ${ }^{80}$ but a formal evaluation of its benefit in BU is not yet available. The use of traditional gauze dressings has been associated with significantly higher infection rates compared to moistretentive dressings in wounds of various origins, but this has not been investigated in BU. ${ }^{13}$ Modern dressing materials such as hydrocolloids may decrease time to healing and improve patient comfort, ${ }^{81-83}$ but again there are no published data relating to their use in BU. The frequency of dressing change depends on the amount of exudate, but routinely it is daily for BU.

The affected part is bandaged and splinted in the best anti-deformity position when on the limbs, avoiding the immobilization of adjacent body parts that are not involved. A carefully applied light elastic bandage provides light compression to decrease edema and hypertrophic scar formation being careful not to restrict movements or to be too tight.

In a recent study in Ghana and Benin, there appeared to be a general understanding of wound assessment, but a large variety of different topical antiseptics were applied in some treatment centers. Gauze was the main dressing type. The standard of wound care differed significantly between health care personnel and between institutions, and adherence to the WHO guidelines was low. ${ }^{13}$

\section{Topical treatment}

M. ulcerans is predominantly a skin infection; hence, topical treatment would be an obvious choice in resource-limited settings. Many types of topical treatment have been reported, but only topical nitrogen-oxide-releasing creams have been shown to be of benefit in a controlled clinical trial in ulcers up to $15 \mathrm{~cm}$ in diameter. ${ }^{84}$ In vitro nitric oxide kills $M$. ulcerans, ${ }^{85}$ but its potential role in treatment in the context of antibiotic treatment is not known. Topical treatment with phenytoin powder has been reported to aid wound healing, and it is believed to stimulate fibrogenesis. ${ }^{86}$ Neither of these agents is currently available routinely.

Taking advantage of the temperature sensitivity of $M$. ulcerans, localized application of heat to lesions has been 
reported to help healing with or without surgery in a few cases. ${ }^{87-89}$ However, others have found this treatment ineffective, and the sophisticated nature of the devices and dressings as well as the discomfort prevent it from being used routinely in a tropical setting.

Although hyperbaric oxygen at partial pressures of $2.5 \mathrm{kPa}$ administered to mice infected with M. ulcerans appeared to be beneficial compared with controls, its effectiveness in human beings needs to be demonstrated in controlled trials and the cost of such treatment may prevent its use in clinical practice..$^{90,91}$

These strategies are still experimental and have not been translated into clinical practice.

In rural African communities, most patients first seek advice from traditional healers and receive treatment with unknown compounds contained in traditional topical applications. An example is the application of plant leaves. ${ }^{92}$ None of the traditional topical agents have been shown to be beneficial, and some may be harmful predisposing sometimes to secondary bacterial infection and osteomyelitis.

\section{Secondary infection}

Secondary infection can be life threatening, but it is surprisingly uncommon considering the low standard of wound care in some endemic countries. Recently, secondary infections of BU lesions were shown to be with organisms such as Staphylococcus aureus and Pseudomonas aeruginosa before treatment, $P$. aeruginosa during treatment, and $P$. aeruginosa or Proteus miriabilis after treatment. ${ }^{93}$ Notably, $78 \%$ of the patients with BU on admission in Ghana and Benin used other antibiotics apart from the standard combination of streptomycin and rifampicin, ${ }^{94}$ and $S$. aureus in BU lesions showed a high frequency of resistance to the first-line drugs used in Ghana as well as the presence of methicillin-resistant $S$. aureus. Whole genome sequencing of $S$. aureus isolates from patients with BU and the subsequent analysis of sequencing data using the SeqSphere scheme reveal likely patient-to-patient transmission events in a health care setting in Ghana indicating a need for the implementation of improved hygiene protocols in health care settings, where patients with BU receive wound care. ${ }^{95,96} M R S A$ antibiotic sensitivity data suggest susceptibility to gentamicin and ofloxacin, but these and alternatives such as vancomycin are usually not available or too expensive. Patients managed in outpatient clinics do not routinely receive treatment for secondary infections such as MRSA but nevertheless go on to heal, so it is possible that some of these simply reflect colonization, sometimes in the presence of a paradoxical reaction, rather than pathogenic wound infection. If there are signs of secondary bacterial infection, a swab should be taken for culture, but this cannot always guide individual care. ${ }^{94}$ Dressings like those that release bactericidal nitric oxide $^{84,85}$ or that retain $M$. ulcerans and other wound pathogens such as Dialkyl carbamoyl chloride-coated dressings ${ }^{97}$ may improve healing by reducing the bacterial load in BUs, but these have yet to be tested.

\section{Pain and wound care}

As treatment progresses, sensitivity to pain returns and adequate pain relief should be provided before the dressings on large ulcers are changed. Nerve regeneration during the healing process is proposed as a possible reason for this finding. Pain during wound care was shown to be associated with every manipulation. ${ }^{98}$ By using self-reported pain scales, pain prior to and during wound care is in the midrange, with severe pain (scores $>6$ during treatment) in nearly $30 \%$ of the patients. Factors associated with higher pain scores during treatment were: being male, fear prior to treatment, pain during the night prior to the wound care, and previous pain while cleaning the wound. ${ }^{99,100}$ A standardized pain protocol for BU wound care will be needed to guide health workers in the establishment of the level of pain and guidance in the use of analgesia.

\section{The role of surgery and wound care}

Small ulcers usually heal without surgical intervention, but if surgery is available, timely split-skin grafting can lead to more rapid healing of larger ulcers provided that infection with $M$. ulcerans has been eliminated by antibiotic treatment. Wide surgical removal of infected tissue is no longer necessary to achieve microbiological cure. Very large ulcers may require multiple conservative surgical interventions for debridement of necrotic tissue and to graft new skin. Contractures (Figure 3 ) are treated by surgical release and deformities are corrected by reconstructive surgery. ${ }^{101-103}$ However, the option of surgery may not be readily available or affordable in endemic countries.

In Australia where surgical services are widely available, surgery is offered if it is considered to be beneficial for wound healing (eg, by debridement of extensive tissue necrosis) or to lessen scarring or deformity, also if antibiotics are contraindicated, refused, or not tolerated after $<4$ weeks of total treatment. Sometimes patients request surgery to hasten wound healing. Ideally, antibiotics are administered for 4 weeks before surgery, but in practice this is variable. Surgical debridement is usually followed by primary or 
secondary wound closure, by direct suture, free skin graft, or flap. ${ }^{3}$

\section{Disability, stigmatization, and rehabilitation}

$\mathrm{BU}$ is endemic in poor rural communities with limited access to health care especially in Africa. Patients tend to report to the hospital late in the course of the disease, some with extensive and long-standing ulcers with joint and bone involvement leading to fibrosis, scarring, calcification, and contractures with permanent disabilities (Figure 3). Fixed joint deformities are a consequence of BU particularly when the lesion is close to a joint. ${ }^{9}$ For late-presenting lesions, 33.6\% in Ghana ${ }^{103,104}$ and $26.0 \%$ in Cote d'Ivoire ${ }^{105}$ developed disabilities. Factors associated with late presentation include herbal treatment, ${ }^{106}$ superstitious beliefs, and fear of surgery. ${ }^{107}$ In some cases, with advance lesions that have affected joints and bones, amputation may be the only option for these lesions to heal. ${ }^{108}$ Lesions in the head and neck region particularly could result in visual impairment and extensive facial scars that have negative impact on the patients including their self-confidence. ${ }^{103}$ These physical limitations together with high cost of patient management may result in psychosocial and economic problems. ${ }^{109}$ Early case detection, optimization of treatment, and rehabilitation are the major objectives to reduce morbidity and disability. ${ }^{110}$

\section{Functional limitations}

Functional limitations are frequent and various methods have been developed to assess them following BUD. Reduced range of movement of one or more joints hampering daily activities affected $27 \%$ of patients in one study. ${ }^{110} \mathrm{~A} \mathrm{BU}$ functional limitation scoring system has been developed to assess the nature and severity of limitation, ${ }^{111}$ which is helpful in clinical trials of treatment. ${ }^{112}$ The BU functional limitation scoring system comprises a list of daily activities that were investigated in patients with BU in Benin and Ghana following treatment. Using this system, 362 (57\%) of 638 patients in Benin and Ghana had persistent functional limitation after 4 years. ${ }^{12}$ The average limitation score was variable with $31 \%$ in Benin and $15 \%$ in Ghana. The mean limitation score in 65 patients without visible contractures was $13 \%$, whereas in 23 patients with visible contractures or an amputation it was $>50 \%$. Using this approach, higher risk for functional limitation was associated with larger lesions at presentation, lesions on a joint, muscular atrophy, and amputation. ${ }^{107}$

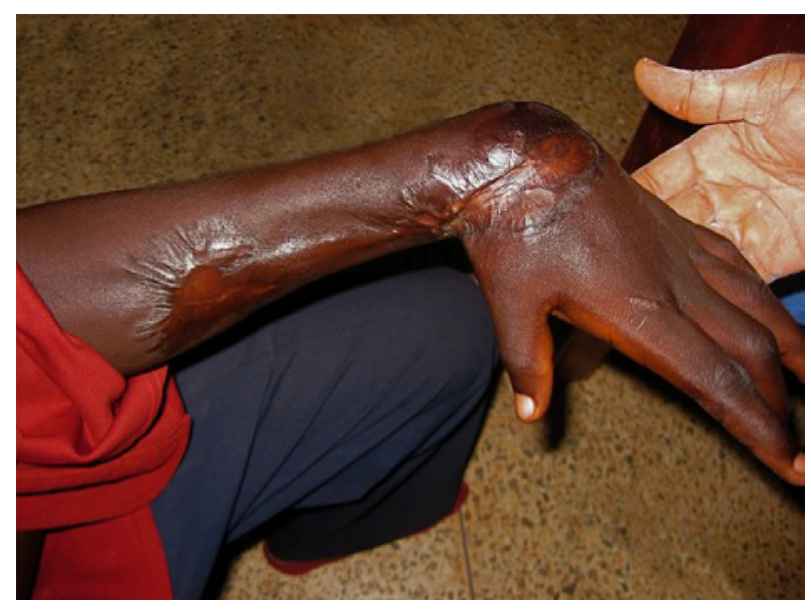

Figure 3 Healed $B U$ of the left upper limb with extensive scarring and flexion deformity at the wrist.

Note: Courtesy of BU photo library of Agogo Presbyterian Hospital, Agogo, Ghana. Abbreviation: BU, Buruli ulcer.

\section{Prevention of disability}

Disability with BU can be prevented or minimized through early diagnosis, antibiotic treatment, and surgical excision, together with adequate management of skin, soft tissues (tendon, ligament, muscle), and joints during the woundhealing process. Prevention of disability in BU is a process by which actions are taken to prevent or minimize complications that can cause disability. This may include physical, socioeconomic, psychological, spiritual, environmental, and personal areas. ${ }^{113}$ To address this, the WHO has developed prevention of disability programs to be implemented in endemic countries.

Limitation of movement is assessed before treatment, and physiotherapy services are provided at patient care centers. Where a physiotherapy center is not available, simple exercises can be administered by health care workers with minimal training ${ }^{114}$ or by the patient and family. This may play an important role in prevention of fixed joint deformities.

These activities include training in wound and skin care, reducing swelling in edema lesions by elevating the affected part, managing scars by lubrication and massage, and improving mobility through exercise. ${ }^{72,115}$

Poor implementation of such programs results in patients developing physical disabilities such as scarring, contractures, deformities, and sometimes requiring amputation ${ }^{116}$ or are otherwise left with functional limitations. ${ }^{106,107,110}$ The WHO advocates for increased public education through outreach programs and early treatment for improvement in disease outcome. 


\section{Participation restrictions and stigma}

Stigmatization against new and former patients is still a challenge for BU control efforts. ${ }^{117}$ Superstition and religious beliefs on the cause, visible signs, and fear of contracting $\mathrm{BU}$ are contributors to this stigma. ${ }^{118} \mathrm{~A}$ study conducted to analyze the long-term psychosocial effects on former patients with BU showed that $50.8 \%$ of cured patients attributed the disease to witchcraft and $2.5 \%$ of patients were rejected by their families. ${ }^{119}$ Socially, the disease impacts on employment, education, relationships, and visiting public places, but most restrictions are related to employment. ${ }^{17,120,121}$

Stigmatization is perceived by former patients with $\mathrm{BU}$ even years after healing. ${ }^{122}$ Participation restrictions are defined as "any problem an individual may experience in involvement in life situations". ${ }^{123}$ Former patients with BU may be reviewed using the participation scale for assessing participation restriction and the Explanatory Model Interview Catalog to measure perceived stigma. Participation restrictions were experienced by $47 \%$ of patients in Ghana and Benin with the important predictors of participation restrictions being female sex, perceived stigma, functional limitations, and larger lesions. ${ }^{17,120,124}$ However, patients who report early with smaller lesions and receive 8 weeks of antibiotics therapy have good quality of life in the long term. ${ }^{25}$

\section{Search strategy and selection strategy}

We searched PubMed with the search terms: "Buruli ulcer", "Mycobacterium ulcerans", "mycobacterial skin ulcers" and in association with the terms "wound care", "antibiotic treatment" "surgery", "topical treatment", and "stigmatization", "rehabilitation", "functional limitation", "stigma", "participation restriction", and "pain". In some instances, data published on the WHO website (http://www.who.int/buruli) were included. ${ }^{114}$ We performed a nonsystematic review of the results of our searches and selected articles relevant to the aims of this review.

\section{Conclusion}

Extensive progress has been made in our understanding of the pathogenesis and management of $\mathrm{BU}$ in the past two decades with significant improvement in both the number of patients receiving treatment and the incidence of recurrence since the introduction of antibiotic therapy. As best outcomes across the board are associated with early presentation and treatment, increased public education through outreach programs is greatly encouraged by the WHO. However, there remains room for improvement in the antibiotic regimen itself and gaps in knowledge as to why wounds of similar size heal at different rates. This may partly be due to wound care practices, and improved adherence to the WHO standardized wound care protocols would probably have a significant impact on healing. However, further research is needed to elucidate the reasons for slow healing including the role of paradoxical reactions.

There is evidence that stigmatization and participation restriction still exist for patients with $\mathrm{BU}$, and it is vital that education is used to reduce these effects.

\section{Disclosure}

The authors report no conflicts of interest in this work.

\section{References}

1. WHO [webpage on the Internet]. Buruli Ulcer (Mycobacterium ulcerans Infection). Fact Sheet $N^{\circ} 199$. Geneva: WHO Health Organisation; 2016. Available from: http://www.who.int/mediacentre/factsheets/ fs199/en/. Accessed January 30, 2016.

2. Demangel C, Stinear TP, Cole ST. Buruli ulcer: reductive evolution enhances pathogenicity of Mycobacterium ulcerans. Nat Rev Microbiol. 2009;7(1):50-60.

3. WHO. Treatment of Mycobacterium ulcerans Disease (Buruli Ulcer): Guidance for Health Workers. Geneva: World Health Organization; 2012. (WHO/HTM/NTD/IDM/2012.1).

4. Bratschi MW, Bolz M, Minyem JC, et al. Geographic distribution, age pattern and sites of lesions in a cohort of Buruli ulcer patients from the Mape Basin of Cameroon. PLoS Negl Trop Dis. 2013;7(6):e2252.

5. Bratschi MW, Ruf MT, Andreoli A, et al. Mycobacterium ulcerans persistence at a village water source of Buruli ulcer patients. PLoS Negl Trop Dis. 2014;8(3):e2756.

6. Etuaful S, Carbonnelle B, Grosset J, et al. Efficacy of the combination rifampin-streptomycin in preventing growth of Mycobacterium ulcerans in early lesions of Buruli ulcer in humans. Antimicrob Agents Chemother. 2005;49(8):3182-3186.

7. Phanzu DM, Suykerbuyk P, Imposo DB, et al. Effect of a control project on clinical profiles and outcomes in buruli ulcer: a before/after study in Bas-Congo, Democratic Republic of Congo. PLoS Negl Trop Dis. 2011;5(12):e1402.

8. Sarfo FS, Phillips R, Asiedu K, et al. Clinical efficacy of combination of rifampin and streptomycin for treatment of Mycobacterium ulcerans disease. Antimicrob Agents Chemother. 2010;54(9):3678-3685.

9. Vincent QB, Ardant MF, Adeye A, et al. Clinical epidemiology of laboratory-confirmed Buruli ulcer in Benin: a cohort study. Lancet Glob Health. 2014;2(7):e422-e430.

10. Sarfo FS, Phillips RO, Zhang J, et al. Kinetics of mycolactone in human subcutaneous tissue during antibiotic therapy for Mycobacterium ulcerans disease. BMC Infect Dis. 2014;14:202.

11. Beissner M, Arens N, Wiedemann F, et al. Treatment outcome of patients with Buruli ulcer disease in Togo. PLoS Negl Trop Dis. 2015;9(10):e0004170.

12. O'Brien DP, Hughes AJ, Cheng AC, et al. Outcomes for Mycobacterium ulcerans infection with combined surgery and antibiotic therapy: findings from a south-eastern Australian case series. Med J Aust. 2007; 186(2):58-61.

13. Velding K, Klis SA, Abass KM, Tuah W, Stienstra Y, van der Werf T. Wound care in buruli ulcer disease in Ghana and Benin. Am J Trop Med Hyg. 2014;91(2):313-318. 
14. Huygen K, Adjei O, Affolabi D, et al. Buruli ulcer disease: prospects for a vaccine. Med Microbiol Immunol. 2009;198(2):69-77.

15. WHO [webpage on the Internet]. Global Health Observatory Data Repository. Millennium Development Goals 6: Neglected Tropical Diseases [App]. 2014. Available from: http://apps.who.int/neglected_ diseases/ntddata/buruli/buruli.html. Accessed January 30, 2016.

16. WHO [webpage on the Internet]. Buruli Ulcer Endemic Countries. 2016. Available from: http://www.who.int/buruli/country/en/. Accessed January 30, 2016.

17. Portaels F, Elsen P, Guimaraes-Peres A, Fonteyne PA, Meyers WM. Insects in the transmission of Mycobacterium ulcerans infection. Lancet. 1999;353(9157):986.

18. Merritt RW, Walker ED, Small PL, et al. Ecology and transmission of Buruli ulcer disease: a systematic review. PLoS Negl Trop Dis. 2010;4(12):e911.

19. Marion E, Eyangoh S, Yeramian E, et al. Seasonal and regional dynamics of M. ulcerans transmission in environmental context: deciphering the role of water bugs as hosts and vectors. PLoS Negl Trop Dis. 2010;4(7):e731.

20. Carson C, Lavender CJ, Handasyde KA, et al. Potential wildlife sentinels for monitoring the endemic spread of human buruli ulcer in South-East Australia. PLoS Negl Trop Dis. 2014;8(1):e2668.

21. Fyfe JA, Lavender CJ, Handasyde KA, et al. A major role for mammals in the ecology of Mycobacterium ulcerans. PLoS Negl Trop Dis. 2010;4(8):e791.

22. Johnson PD, Azuolas J, Lavender CJ, et al. Mycobacterium ulcerans in mosquitoes captured during outbreak of Buruli ulcer, southeastern Australia. Emerg Infect Dis. 2007;13(11):1653-1660.

23. Portaels F, Silva MT, Meyers WM. Buruli ulcer. Clin Dermatol. 2009;27(3):291-305.

24. Pommelet V, Vincent QB, Ardant MF, et al. Findings in patients from Benin with osteomyelitis and polymerase chain reactionconfirmed Mycobacterium ulcerans infection. Clin Infect Dis. 2014;59(9):1256-1264.

25. Capela C, Sopoh GE, Houezo JG, et al. Clinical epidemiology of Buruli ulcer from Benin (2005-2013): effect of time-delay to diagnosis on clinical forms and severe phenotypes. PLoS Negl Trop Dis. 2015;9(9):e0004005.

26. Portaels F, Agular J, Fissette K, et al. Direct detection and identification of Mycobacterium ulcerans in clinical specimens by PCR and oligonucleotide-specific capture plate hybridization. J Clin Microbiol. 1997;35(5):1097-1100.

27. Phillips R, Horsfield C, Kuijper S, et al. Sensitivity of PCR targeting the IS2404 insertion sequence of Mycobacterium ulcerans in an assay using punch biopsy specimens for diagnosis of Buruli ulcer. $J$ Clin Microbiol. 2005;43(8):3650-3656.

28. Herbinger KH, Adjei O, Awua-Boateng NY, et al. Comparative study of the sensitivity of different diagnostic methods for the laboratory diagnosis of Buruli ulcer disease. Clin Infect Dis. 2009;48(8):1055-1064.

29. Phillips RO, Sarfo FS, Osei-Sarpong F, et al. Sensitivity of PCR targeting Mycobacterium ulcerans by use of fine-needle aspirates for diagnosis of Buruli ulcer. J Clin Microbiol. 2009;47(4):924-926.

30. Ross BC, Johnson PD, Oppedisano F, et al. Detection of Mycobacterium ulcerans in environmental samples during an outbreak of ulcerative disease. Appl Environ Microbiol. 1997;63(10):4135-4138.

31. Stinear T, Ross BC, Davies JK, et al. Identification and characterization of IS2404 and IS2606: two distinct repeated sequences for detection of Mycobacterium ulcerans by PCR. J Clin Microbiol. 1999;37(4):1018-1023.

32. Guimaraes-Peres A, Portaels F, de Rijk P, et al. Comparison of two PCRs for detection of Mycobacterium ulcerans. J Clin Microbiol. 1999;37(1):206-208.

33. Stienstra Y, van der Werf TS, Guarner J, et al. Analysis of an IS2404-based nested PCR for diagnosis of Buruli ulcer disease in regions of Ghana where the disease is endemic. J Clin Microbiol. 2003;41(2):794-797.
34. Herbinger KH, Beissner M, Huber K, et al. Efficiency of fineneedle aspiration compared with other sampling techniques for laboratory diagnosis of Buruli ulcer disease. J Clin Microbiol. 2010;48(10):3732-3734.

35. Eddyani M, Fraga AG, Schmitt F, et al. Fine-needle aspiration, an efficient sampling technique for bacteriological diagnosis of nonulcerative Buruli ulcer. J Clin Microbiol. 2009;47(6):1700-1704.

36. Hayman J, McQueen A. The pathology of Mycobacterium ulcerans infection. Pathology. 1985;17(4):594-600.

37. Connor DH, Lunn HF. Mycobacterium ulcerans infection (with comments on pathogenesis). Int J Lepr. 1965;33((3): suppl):698-709.

38. George KM, Chatterjee D, Gunawardana G, et al. Mycolactone: a polyketide toxin from Mycobacterium ulcerans required for virulence. Science. 1999;283(5403):854-857.

39. Stinear TP, Mve-Obiang A, Small PL, et al. Giant plasmid-encoded polyketide synthases produce the macrolide toxin of Mycobacterium ulcerans. Proc Natl Acad Sci U S A. 2004;101(5):1345-1349.

40. Mve-Obiang A, Lee RE, Portaels F, Small PL. Heterogeneity of mycolactones produced by clinical isolates of Mycobacterium ulcerans: implications for virulence. Infect Immun. 2003;71(2):774-783.

41. Snyder DS, Small PL. Uptake and cellular actions of mycolactone, a virulence determinant for Mycobacterium ulcerans. Microb Pathog. 2003;34(2):91-101.

42. Guenin-Mace L, Veyron-Churlet R, Thoulouze MI, et al. Mycolactone activation of Wiskott-Aldrich syndrome proteins underpins Buruli ulcer formation. J Clin Invest. 2013;123(4):1501-1512.

43. Simmonds RE, Lali FV, Smallie T, Small PL, Foxwell BM. Mycolactone inhibits monocyte cytokine production by a posttranscriptional mechanism. J Immunol. 2009;182(4):2194-2202.

44. Coutanceau E, Decalf J, Martino A, et al. Selective suppression of dendritic cell functions by Mycobacterium ulcerans toxin mycolactone. J Exp Med. 2007;204(6):1395-1403.

45. Boulkroun S, Guenin-Mace L, Thoulouze MI, et al. Mycolactone suppresses $\mathrm{T}$ cell responsiveness by altering both early signaling and posttranslational events. J Immunol. 2010;184(3):1436-1444.

46. Guenin-Mace L, Carrette F, Asperti-Boursin F, et al. Mycolactone impairs $\mathrm{T}$ cell homing by suppressing microRNA control of L-selectin expression. Proc Natl Acad Sci U S A. 2011;108(31): 12833-12838.

47. Hall B, Simmonds R. Pleiotropic molecular effects of the Mycobacterium ulcerans virulence factor mycolactone underlying the cell death and immunosuppression seen in Buruli ulcer. Biochem Soc Trans. 2014;42(1):177-183.

48. Ogbechi J, Ruf MT, Hall BS, et al. Mycolactone-dependent depletion of endothelial cell thrombomodulin is strongly associated with fibrin deposition in Buruli ulcer lesions. PLoS Pathog. 2015;11(7):e1005011.

49. Sarfo FS, Phillips R, Wansbrough-Jones M, Simmonds RE. Recent advances: role of mycolactone in the pathogenesis and monitoring of Mycobacterium ulcerans infection/Buruli ulcer disease. Cell Microbiol. 2016;18(1):17-29.

50. Havel A, Pattyn SR. Activity of rifampicin on Mycobacterium ulcerans. Ann Soc Belg Med Trop. 1975;55(2):105-108.

51. Thangaraj HS, Adjei O, Allen BW, et al. In vitro activity of ciprofloxacin, sparfloxacin, ofloxacin, amikacin and rifampicin against Ghanaian isolates of Mycobacterium ulcerans. J Antimicrob Chemother. 2000;45(2):231-233.

52. Portaels F, Traore H, De Ridder K, Meyers WM. In vitro susceptibility of Mycobacterium ulcerans to clarithromycin. Antimicrob Agents Chemother. 1998;42(8):2070-2073.

53. Bentoucha A, Robert J, Dega H, Lounis N, Jarlier V, Grosset J. Activities of new macrolides and fluoroquinolones against Mycobacterium ulcerans infection in mice. Antimicrob Agents Chemother. 2001;45(11):3109-3112.

54. Dega H, Robert J, Bonnafous P, Jarlier V, Grosset J. Activities of several antimicrobials against Mycobacterium ulcerans infection in mice. Antimicrob Agents Chemother. 2000;44(9):2367-2372. 
55. Stanford JL, Phillips I. Rifampicin in experimental Mycobacterium ulcerans infection. J Med Microbiol. 1972;5(1):39-45.

56. Marsollier L, Honore N, Legras P, et al. Isolation of three Mycobacterium ulcerans strains resistant to rifampin after experimental chemotherapy of mice. Antimicrob Agents Chemother. 2003;47(4):1228-1232.

57. Chauty A, Ardant MF, Adeye A, et al. Promising clinical efficacy of streptomycin-rifampin combination for treatment of buruli ulcer (Mycobacterium ulcerans disease). Antimicrob Agents Chemother. 2007;51(11):4029-4035.

58. Nienhuis WA, Stienstra Y, Thompson WA, et al. Antimicrobial treatment for early, limited Mycobacterium ulcerans infection: a randomised controlled trial. Lancet. 2010;375(9715):664-672.

59. Phillips RO, Sarfo FS, Abass MK, et al. Clinical and bacteriological efficacy of rifampin-streptomycin combination for two weeks followed by rifampin and clarithromycin for six weeks for treatment of Mycobacterium ulcerans disease. Antimicrob Agents Chemother. 2014;58(4):2488.

60. O'Brien DP, Comte E, Ford N, Christinet V, du Cros P. Moxifloxacin for Buruli ulcer/HIV coinfected patients: kill two birds with one stone? AIDS. 2013;27(14):2177-2179.

61. Ruf MT, Chauty A, Adeye A, et al. Secondary Buruli ulcer skin lesions emerging several months after completion of chemotherapy: paradoxical reaction or evidence for immune protection? PLoS Negl Trop Dis. 2011;5(8):e1252.

62. Nienhuis WA, StienstraY, Abass KM, et al. Paradoxical responses after start of antimicrobial treatment in Mycobacterium ulcerans infection. Clin Infect Dis. 2012;54(4):519-526.

63. Wanda F, Nkemenang P, Ehounou G, et al. Clinical features and management of a severe paradoxical reaction associated with combined treatment of Buruli ulcer and HIV co-infection. BMC Infect Dis. 2014;14:423.

64. O'Brien DP, Robson ME, Callan PP, McDonald AH. "Paradoxical" immune-mediated reactions to Mycobacterium ulcerans during antibiotic treatment: a result of treatment success, not failure. Med J Aust. 2009;191(10):564-566.

65. Mattie H, Craig WA, Pechere JC. Determinants of efficacy and toxicity of aminoglycosides. J Antimicrob Chemother. 1989;24(3):281-293.

66. Selimoglu E. Aminoglycoside-induced ototoxicity. Curr Pharm Des. 2007;13(1):119-126.

67. Klis S, Stienstra Y, Phillips RO, Abass KM, Tuah W, van der Werf TS. Long term streptomycin toxicity in the treatment of Buruli ulcer: follow-up of participants in the BURULICO drug trial. PLoS Negl Trop Dis. 2014;8(3):e2739.

68. Guthrie OW. Aminoglycoside induced ototoxicity. Toxicology. 2008;249(2-3):91-96.

69. WHO [webpage on the Internet]. Wound Management. 2015. Available from: http://www.who.int/buruli/information/publications/BU-5PODinterventions-2.pdf?ua=1. Assessed October 7, 2015.

70. Lee JC, Kandula S, Sherber NS. Beyond wet-to-dry: a rational approach to treating chronic wounds. Eplasty. 2009;9:e14.

71. WHO, Macdonald JM, Geyer MJ. Wound and Lymphoedema Management. Geneva: World Health Organization; 2010.

72. Lehman L, Simonet V, Saunderson P, Agbenorku P. Buruli Ulcer: Prevention of Disability (POD). Geneva: World Health Organization; 2006.

73. Welch JS, Bunting TG. The cytotoxic effects of commonly used topical antimicrobial agents on human fibroblasts and keratinocytes. JTrauma. 1993;35(3):491-492.

74. Thomas GW, Rael LT, Bar-Or R, et al. Mechanisms of delayed wound healing by commonly used antiseptics. J Trauma. 2009;66(1):82-90.

75. Wilson JR, Mills JG, Prather ID, Dimitrijevich SD. A toxicity index of skin and wound cleansers used on in vitro fibroblasts and keratinocytes. Adv Skin Wound Care. 2005;18(7):373-378.

76. Sarabahi S. Recent advances in topical wound care. Indian J Plast Surg. 2012;45(2):379-387.

77. O’Meara S, Cullum N, Majid M, Sheldon T. Systematic reviews of wound care management: (3) antimicrobial agents for chronic wounds; (4) diabetic foot ulceration. Health Technol Assess. 2000;4(21):1-237.
78. Bradley M, Cullum N, Nelson EA, Petticrew M, Sheldon T, Torgerson D. Systematic reviews of wound care management: (2). Dressings and topical agents used in the healing of chronic wounds. Health Technol Assess. 1999;3(17 pt 2):1-35.

79. Amoakoh HB, Aikins M. Household cost of out-patient treatment of Buruli ulcer in Ghana: a case study of Obom in Ga South Municipality. BMC Health Serv Res. 2013;13:507.

80. Brown A, Yorke M. Drawtex: breaking the vicious circle of cellular and molecular imbalances. Br J Community Nurs. 2013;(suppl): S42, S44, S46-S44.

81. Davies P, Rippon M. Comparison of Foam and Hydrocolloid Dressings in the Management of Wounds: A Review of the Published Literature. 2010. Available from: http://www.worldwidewounds.com/2010/July/ DaviesRippon/DaviesRippon.html. Accessed January 30, 2016.

82. Martini L, Reali UM, Borgognoni L, Brandani P, Andriessen A. Comparison of two dressings in the management of partial-thickness donor sites. J Wound Care. 1999;8(9):457-460.

83. Chang KW, Alsagoff S, Ong KT, Sim PH. Pressure ulcers - randomised controlled trial comparing hydrocolloid and saline gauze dressings. Med J Malaysia. 1998;53(4):428-431.

84. Phillips R, Adjei O, Lucas S, Benjamin N, Wansbrough-Jones M. Pilot randomized double-blind trial of treatment of Mycobacterium ulcerans disease (Buruli ulcer) with topical nitrogen oxides. Antimicrob Agents Chemother. 2004;48(8):2866-2870.

85. Phillips R, Kuijper S, Benjamin N, Wansbrough-Jones M, Wilks M, Kolk AH. In vitro killing of Mycobacterium ulcerans by acidified nitrite. Antimicrob Agents Chemother. 2004;48(8):3130-3132.

86. Adjei O, Evans MR, Asiedu A. Phenytoin in the treatment of Buruli ulcer. Trans R Soc Trop Med Hyg. 1998;92(1):108-109.

87. Reid IS. Mycobacterium ulcerans infection: a report of 13 cases at the Port Moresby General Hospital, Papua. Med JAust. 1967;1(9):427-431.

88. Glynn PJ. The use of surgery and local temperature elevation in Mycobacterium ulcerans infection. Aust N Z J Surg. 1972;41(4):312-317.

89. Meyers WM, Shelly WM, Connor DH. Heat treatment of Mycobacterium ulcerans infections without surgical excision. Am J Trop Med Hyg. 1974;23(5):924-929.

90. Krieg RE, Wolcott JH, Confer A. Treatment of Mycobacterium ulcerans infection by hyperbaric oxygenation. Aviat Space Environ Med. 1975;46(10):1241-1245.

91. Krieg RE, Wolcott JH, Meyers WM. Mycobacterium ulcerans infection: treatment with rifampin, hyperbaric oxygenation, and heat. Aviat Space Environ Med. 1979;50(9):888-892.

92. Landier J, Boisier P, Fotso Piam F, et al. Adequate wound care and use of bed nets as protective factors against Buruli ulcer: results from a case control study in Cameroon. PLoS Negl Trop Dis. 2011;5(11):e1392.

93. Yeboah-Manu D, Kpeli GS, Ruf MT, et al. Secondary bacterial infections of buruli ulcer lesions before and after chemotherapy with streptomycin and rifampicin. PLoS Negl Trop Dis. 2013;7(5):e2191.

94. Barogui YT, Klis S, Bankole HS, et al. Towards rational use of antibiotics for suspected secondary infections in buruli ulcer patients. PLoS Negl Trop Dis. 2013;7(1):e2010.

95. Amissah NA, Chlebowicz MA, Ablordey A, et al. Molecular characterization of Staphylococcus aureus isolates transmitted between patients with Buruli ulcer. PLoS Negl Trop Dis. 2015;9(9):e0004049.

96. Amissah NA, Glasner C, Ablordey A, et al. Genetic diversity of Staphylococcus aureus in Buruli ulcer. PLoS Negl Trop Dis. 2015;9(2):e0003421.

97. Geroult S, Phillips RO, Demangel C. Adhesion of the ulcerative pathogen Mycobacterium ulcerans to DACC-coated dressings. JWound Care. 2014;23(8):417-424.

98. de Jong A, Baartmans M, Bremer M, et al. Reliability, validity and clinical utility of three types of pain behavioural observation scales for young children with burns aged 0-5 years. Pain. 2010;150(3):561-567.

99. Alferink M, de Zeeuw J, Sopoh G, et al. Pain associated with wound care treatment among Buruli ulcer patients from Ghana and Benin. PLoS One. 2015;10(6):e0119926. 
100. de Zeeuw J, Alferink M, Barogui YT, et al. Assessment and treatment of pain during treatment of Buruli ulcer. PLoS Negl Trop Dis. 2015;9(9):e0004076.

101. Agbenorku P, Donwi IK, Kuadzi P, Saunderson P. Buruli ulcer: treatment challenges at three centres in Ghana. Re Dai Yi Xue Za Zhi. 2012;2012:371915.

102. Agbenorku P, Agbenorku M, Amankwa A, Tuuli L, Saunderson P. Factors enhancing the control of Buruli ulcer in the Bomfa communities, Ghana. Trans R Soc Trop Med Hyg. 2011;105(8):459-465.

103. Agbenorku P. Multicenter study of buruli ulcer disabilities in the head and neck region. Plast Surg Int. 2011;2011:647418.

104. Agbenorku P, Edusei A, Agbenorku M, et al. Buruli-ulcer induced disability in Ghana: a study at Apromase in the Ashanti Region. Plast Surg Int. 2012;2012:752749.

105. Agbenorku P, Agbenorku M, Saunderson P, Lehman L. The benefits of a combination of surgery and chemotherapy in the management of Buruli ulcer patients. J Sci Technol. 2006;26(2):6-10.

106. Stienstra Y, van Roest MH, van Wezel MJ, et al. Factors associated with functional limitations and subsequent employment or schooling in Buruli ulcer patients. Trop Med Int Health. 2005;10(12):1251-1257.

107. Barogui Y, Johnson RC, van der Werf TS, et al. Functional limitations after surgical or antibiotic treatment for Buruli ulcer in Benin. Am J Trop Med Hyg. 2009;81(1):82-87.

108. van der Werf TS, Stienstra Y, Johnson RC, et al. Mycobacterium ulcerans disease. Bull World Health Organ. 2005;83(10):785-791.

109. Asiedu K, Etuaful S. Socioeconomic implications of Buruli ulcer in Ghana: a three-year review. Am JTrop Med Hyg. 1998;59(6):1015-1022.

110. Schunk M, Thompson W, Klutse E, et al. Outcome of patients with buruli ulcer after surgical treatment with or without antimycobacterial treatment in Ghana. Am J Trop Med Hyg. 2009;81(1):75-81.

111. Stienstra Y, Dijkstra PU, Guedenon A, et al. Development of a questionnaire assessing Buruli ulcer-induced functional limitation. $\mathrm{Am} \mathrm{J}$ Trop Med Hyg. 2004;70(3):318-322.

112. Stienstra Y, Dijkstra PU, Van Wezel MJ, et al. Reliability and validity of the Buruli ulcer functional limitation score questionnaire. Am J Trop Med Hyg. 2005;72(4):449-452.

113. World Health Organisation (WHO) [webpage on the Internet]. Concepts of Prevention of Disability and Rehabilitation. 2015. Available from: http://www.who.int/buruli/information/publications/pod/en/. Assessed October 7, 2015.
114. WHO [webpage on the Internet]. WHO Publications on Buruli Ulcer Available from: http:/www.who.int/buruli/information/publications/ en/. Accessed January 30, 2016.

115. WHO [webpage on the Internet]. Simonet V, editor. Prevention of Disability in Buruli Ulcer: Basic Rehabilitation. A Practical Field Guide. Geneva: World Health Organization; 2008. Available from: http://whqlibdoc.who.int/hq/2008/WHO_HTM_NTD_IDM_ GBUI_2008.1_eng.pdf. Accessed January 30, 2016.

116. Ellen DE, Stienstra Y, Teelken MA, Dijkstra PU, van der Graaf WT, van der Werf TS. Assessment of functional limitations caused by Mycobacterium ulcerans infection: towards a Buruli ulcer functional limitation score. Trop Med Int Health. 2003;8(1):90-96.

117. de Zeeuw J, Omansen TF, Douwstra M, et al. Persisting social participation restrictions among former buruli ulcer patients in Ghana and Benin. PLoS Negl Trop Dis. 2014;8(11):e3303.

118. Stienstra Y, van der Graaf WT, Asamoa K, van der Werf TS. Beliefs and attitudes toward Buruli ulcer in Ghana. Am J Trop Med Hyg. 2002;67(2):207-213.

119. Kpadonou TG, Alagnide E, Azanmasso H, et al. Psychosocioprofessional and familial becoming of formers Buruli ulcer patients in Benin. Ann Phys Rehabil Med. 2013;56(7-8):515-526.

120. de Zeeuw J, Douwstra M, Omansen TF, et al. Psychometric properties of the participation scale among former buruli ulcer patients in Ghana and Benin. PLoS Negl Trop Dis. 2014;8(11):e3254.

121. Renzaho AM, Woods PV, Ackumey MM, Harvey SK, Kotin J. Community-based study on knowledge, attitude and practice on the mode of transmission, prevention and treatment of the Buruli ulcer in Ga West District, Ghana. Trop Med Int Health. 2007;12(3):445-458.

122. Adamba C, Owusu AY. Burden of Buruli ulcer: how affected households in a Ghanaian district cope. Afr Study Monogr. 2011;32(1): $1-23$.

123. World Health Organization (WHO). International Classification of Functioning, Disability and Health: ICF. Geneva: World Health Organization; 2001.

124. Hamzat TK, Boakye-Afram B. Health-related quality of life among persons living with Buruli ulcer in Amasaman community, Ga West District Accra, Ghana. Int J Health Sci. 2011;5(1):29-38.

125. Klis S, Ranchor A, Phillips RO, et al. Good quality of life in former Buruli ulcer patients with small lesions: long-term follow-up of the BURULICO trial. PLoS Negl Trop Dis. 2014;8(7):e2964.
Chronic Wound Care Management and Research

\section{Publish your work in this journal}

Chronic Wound Care Management and Research is an international, peer reviewed, open access, online journal publishing original research, reviews, editorials, and commentaries on the causes and management of chronic wounds and the major issues related to chronic wound management. Topics also include chronic wounds as comorbidities to other

\section{Dovepress}

conditions, patient adherence to therapy, and the economic burden of chronic wounds. The manuscript management system is completely online and includes a very quick and fair peer review system, which is all easy to use. Visit http://www.dovepress.com/testimonials.php to read real quotes from published authors. 